\title{
Specific Time Determinism: Circadian Rhythm of Microbial Community Assembly Patterns Under Common Light in Feces of Laying Hens
}

\section{Yu Zhang}

South China Agricultural University College of Animal Science

\section{Lan Sun}

South China Agricultural University

Run Zhu

South China Agricultural University

Shiyu Zhang

South China Agricultural University

Yan Wang

South China Agricultural University

Yinbao Wu

South China Agricultural University

Xindi Liao

South China Agricultural University

Jiandui Mi ( $\nabla$ mijiandui@163.com )

South China Agricultural University College of Animal Science

\section{Research}

Keywords: Feces, Microbiota, Laying hen, Circadian rhythm

Posted Date: January 15th, 2021

DOI: https://doi.org/10.21203/rs.3.rs-146250/v1

License: (1) (1) This work is licensed under a Creative Commons Attribution 4.0 International License. Read Full License 


\section{Abstract}

\section{Background}

As an important part of biological rhythm, the circadian rhythm of the gut microbiota plays a crucial role in host health. However, few studies have determined the associations between the circadian rhythm and gut microbiota in laying hens. The purpose of this experiment was to investigate the circadian rhythm of the feces microbiota in laying hens.

Results

Feces samples were collected from ten laying hens at nine different time points (06:00-12:00-18:00-00:0006:00-12:00-18:00-00:00-06:00) to demonstrate the diurnal oscillation of the feces microbiota. We described the phenomenon of circadian rhythmicity of the feces microbiota in laying hens based on $16 \mathrm{~S}$ rRNA gene sequencing. According to the results, the $a$ and $\beta$ diversity of the feces microbiota fluctuated significantly at different time points. Beta Nearest Taxon Index analysis suggested that assembly strategies of the abundant and rare amplicon sequence variants (ASVs) sub-communities are different. Abundant ASVs preferred disperal limitation (weak selction), whereas rare ASVs randomly formed due to the "non-dominant" fractions. Highly robust circadian fluctuations were found, for instance, in Firmicutes and Proteobacteria, which fluctuated oppositely, and the total ratio remained in a dynamic balance over $48 \mathrm{~h}$. We found that temporal dynamic changes had a significant effect on the relative abundance of the important bacteria in the feces microbiota community by using the random forest algorithm. Eight bacteria, Ruminococcus gnavus, Faecalibacterium, Ruminococcaceae, Enterococcus cecorum, Lachnospiraceae, Clostridium, Clostridiales, and Megamonas, gained rhythmicity. Interestingly, one unexpected finding was the fact that these eight bacteria all belong to Firmicutes. In particular, the microbiota profile appears to favor butyrate production, a common indicator of gut health, potentially through increases in the members of Lachnospiraceae, Faecalibacterium and Ruminococcaceae. The pathways were the functionalities that gained rhythmicity within the microbiota, which belonged to the most abundant functions, including xenobiotic biodegradation and metabolism, carbohydrate metabolism, and amino acid metabolism, which were consistent with the metabolic functions of amino acids and carbohydrates from feed.

\section{Conclusion}

This study has shown that the defecation time could be among the important factors influencing the diversity, proportion, and functions of the feces microbiota community. We also note the potential application of beneficial microbial communities in livestock species, especially in the environment of raising antibiotic-free animals. These results provide references for further exploring the circadian rhythm of the gut microbiota in laying hens.

\section{Background}


The poultry industry is an important component of the world agricultural economy, which provides a vital source of protein for the human diet [1-3], and this proportion is constantly growing [4]. Antibiotics are widely used in poultry farming and have attracted widespread attention due to their abuse, to drug resistance $[5,6]$, to pollution of the ecological environment $[7,8]$ and to the threats to human health $[5,9]$. However, with the general trend of "no resistance breeding", intensive laying hen operations are facing a series of problems, such as a high incidence of fatty liver [10], abdominal fat deposition [11], poor eggshell quality [12], and serious pecking addiction $[13,14]$. There is an urgent need to ensure the health of laying hens and their intestinal health to maintain the sustainable development of the industry.

The intestinal microbial community is a highly complex and diverse ecosystem that plays a crucial role in regulating host metabolism, immune modulation and many other key physiological pathways as well as maintaining the homeostasis and health of the host [15-19]. The diversity of the intestinal microbe microbiota has become a potential indicator of overall host health [20]. Targeted regulation of the intestinal microbiota of laying hens has become a new direction to improve the above problems in laying hens. Most organisms have circadian rhythms in physiology and behavior, which are synchronized with the 24-h light/dark cycle of the earth [21]. As an important part of biological rhythm, circadian rhythm can synchronize with the metabolism of the body [22]. In recent years, researchers have shown an increased interest in the circadian rhythm of the intestinal microbiota. The normal intestinal microecosystem maintains a certain circadian rhythm with rhythmic fluctuations in composition and function, and once the intestinal microbiota is disturbed, it will affect host health $[23,24]$. The circadian rhythm of bacteria was first found in Cyanobacteria cells, and their metabolism level changed with changes in illumination time [25]. However, whether the intestinal microbiota is an important microbial community with a similar circadian rhythm in the life activities of animals remains unclear. By adjusting the illumination time [26, 27] and eating time of mice [28], it was proven that the composition and function of the intestinal microbiota fluctuated over $24 \mathrm{~h}$. Interestingly, the relative abundance of more than $15 \%$ of intestinal microbiota showed periodic fluctuations, including Clostridium and Bacteroides [26]. With the deepening of research on the intestinal microbiota, extensive research has shown that the intestinal microbiota does have a 24-h cycle of circadian rhythm fluctuation. The circadian rhythm plays an important role in the host's physiological metabolism, and the activities of the intestinal microbiota also participate in the host's metabolism [29,30]. Moreover, laying hens are more sensitive to light, which is an important factor affecting circadian rhythm, and have a stronger biological rhythm. A suitable illumination scheme can not only improve the production performance of laying hens but also ensure the health of laying hens [31]. Newborn chicks had different intestinal microbiota communities when exposed to different light conditions (12/12-h light and dark (L/D) and 23/1-h L/D), which was shown in one of the earliest studies to prove that the photoperiod could be used to regulate the intestinal microbiota communities of newly hatched chicks [32]. However, there are no more in-depth studies, as the current studies provide only superficial knowledge. At present, there is a lack of more detailed and specific research on the intestinal microbiota of laying hens. In summary, it is necessary to understand the circadian rhythm of the intestinal microbiota in laying hens and provide guidance for the research and maintenance of the intestinal health of laying hens in the future. 
More than $90 \%$ of feces consists of the microbiota, and the "feces microbiota" includes bacteria (93\%), viruses $(5.8 \%)$, archaea $(0.8 \%)$ and eukaryotes $(0.5 \%)$ [33]. Feces characteristics are a sign of intestinal health, which can reflect the status of the intestinal microbiota to a certain extent [34]. The purpose of this experiment was to explore the circadian rhythm of the feces microbiota in Hy-Line Gray laying hens and to provide basic information and methodological support for future basic research on the circadian rhythm of the intestinal microbiota of laying hens.

\section{Experimental Procedures}

\section{Animals and feeding}

Given their widespread application in the poultry industry, Hy-Line Gray laying hens were selected for this study. The 30-week-old Hy-Line Gray laying hens used in this experiment were all fed under the same feeding environment and dietary conditions in a commercial hatchery (Peng Chang Co., Ltd., Shenzhen, China). A total of ten laying hens with similar weights and laying rates were randomly selected. During the experiment, the laying hens received the same commercial laying hen diet ad libitum. The diet based on corn and soybean meal was designed to meet or exceed the energy requirement of the NRC (2012) (Additional file 1: Table S1). Clean drinking water was also provided at all times. The laying hens were kept in a henhouse maintained at $25^{\circ} \mathrm{C}$ with a 16-h lighting (from 6 a.m. to 10 p.m.) and 8-h dark management schedule, and the experiment was carried out for 2 days. None of the laying hens required antibiotics during the sampling period. It should be noted that the diet, age, weight and feeding environment of all laying hens were consistent during the experiment to minimize the possible impact of these factors.

\section{Feces sample collection}

Fresh feces samples were collected from 06:00-12:00-18:00-00:00-06:00-12:00-

18:00-00:00-06:00 (Zeitgeber times [ZT] 0-48) over $48 \mathrm{~h}$ at nine different time points. At each time point, feces samples were collected within $30 \mathrm{~min}$, and a total of 90 fresh feces samples were obtained. Sterile gloves were worn during feces sample collection, and the anus and surrounding area of the laying hens were cleaned with sterile water and then cotton soaked with $75 \%$ ethanol to minimize the risk of contamination. The feces samples were collected manually in sterile tubes. After collection, the samples were immediately placed in liquid nitrogen and stored at $-80^{\circ} \mathrm{C}$ for further experiments.

\section{DNA extraction and 16S rRNA amplification}

Approximately $200 \mathrm{mg}$ of feces sample was used for total DNA extraction using a QIAamp Power Fecal DNA Kit (Qiagen, Germany) following the manufacturer's instructions. The DNA samples were stored at $-20{ }^{\circ} \mathrm{C}$ until further use. The feces microbiota composition profiles over time were determined by $16 \mathrm{~S}$ rRNA gene sequencing. The $\mathrm{V} 4$ hypervariable regions in DNA samples were amplified by 16S rRNA gene PCR using primers (Forward: 5'- GTGCCAGCMGCCGCGGTAA-3' and Reverse: 5'- 
GGACTACHVGGGTWTCTAAT -3') [35]. PCR amplifications were carried out using $25-\mu \mathrm{L}$ reaction mixtures containing $11 \mu \mathrm{L}$ of PCR-grade water, $10 \mu \mathrm{L}$ of 5' PRIME HotMasterMix, $3 \mu \mathrm{L}$ of DNA template and $0.5 \mu \mathrm{L}$ of each primer (initial concentration: $10 \mu \mathrm{M}$ ). PCR amplifications were performed using the following conditions: predenaturation at $94^{\circ} \mathrm{C}$ for $5 \mathrm{~min} ; 30$ cycles of amplification including denaturation at $94^{\circ} \mathrm{C}$ for $30 \mathrm{~s}$, annealing at $50^{\circ} \mathrm{C}$ for $30 \mathrm{~s}$ and elongation at $72^{\circ} \mathrm{C}$ for $30 \mathrm{~s}$; and final elongation at $72^{\circ} \mathrm{C}$ for 10 min. To ensure the efficiency and accuracy of amplification, quantitative and quality inspection of DNA was carried out using a Qubit nucleic acid quantitative analyzer and agarose gel electrophoresis. The final products were sequenced using the Illumina HiSeq PE250 platform. PCR amplification and sequencing were performed by Novogene Co., Ltd. (Tianjin, China).

\section{Sequence analysis}

Raw reads were obtained from the Illumina HiSeq PE250 platform. Raw reads were uploaded into Quantitative Insights into Microbial Ecology (QIIME2-2020.6) software and quality trimmed. The unqualified reads and sequences with poor splicing effects ( $<25$ mass score and $>225$ bp length) were filtered through quality monitoring to obtain clean reads. Trimmed sequences were clustered using the DADA2 method with amplicon sequence variant (ASV) levels. All clean reads were compared with reference sequences to obtain the final mapped reads. Analysis of alpha diversity (Observed species, Chao 1 and Shannon indexes) was calculated in QIIME. For beta diversity analysis, principle coordinates analysis (PCoA) was used to evaluate the difference in bacterial community structure. The functional prediction of the microbial community was based on Phylogenetic Investigation of Communities by Reconstruction of Unobserved States (PICRUSt) and comparison in the Kyoto Encyclopedia of Genes and Genomes (KEGG) database. To evaluate the community assembly processes, the mean nearest taxon distance (MNTD) taxonomic $\beta$-diversity metrics ( $\beta N T I$ and Bray-Curtisbased Raup-Crick, $\mathrm{RC}_{\text {Bray }}$ ) was calculated as previously described [36]. $|\beta N T| \mid>2$ means that the deterministic process is the main factor to influence the microbial community across all samples. In detail, a $\beta N T I$ with value of $<-2$ suggests homogeneous selection and $>2$ means variable selection. If $|\beta N T| \mid<2$, the $R C_{B r a y}$ should be caculated: (1)

$\mathrm{RC}_{\text {Bray }}>0.95$ means dispersal limitations, (2) $\mathrm{RC}_{\text {Bray }}<-0.95$ reveals homogeneous dispersal, and (3) $\left|\mathrm{RC} \mathrm{B}_{\text {Bray }}\right|$ $<0.95$ indicates "non-dominant" fractions [37, 38]. The interpretation degree of the difference in feces bacterial community structure was analyzed by a linear model, and the significance analysis was conducted by using the substitution test. $P$-values less than 0.05 were considered statistically significant. The machine-learning method random forest (RF) was used to identify the top 30 genera and their functions during the different times.

\section{Construction of a cooccurrence network}

The cooccurrence mode of the dominant genera was constructed based on SparCC's rank correlations of bacterial abundance on the network interface to understand the relationship between predominant genera in the feces samples. The effective cooccurrence events were based on strong and significant correlations between the predominant bacteria. Nodes in the network represented the predominant 
bacteria at the genus and family levels, and the edges indicated the relationship between them. The size of each node was proportional to its degree (the number of connections) in the dataset.

\section{Results}

\section{Over Feces Microbiota Structure in Laying Hens}

Feces samples $(n=90)$ were collected from ten laying hens at nine different time points (06:00-12:0018:00-00:00-06:00-12:00-18:00-00:00-06:00) to investigate the circadian rhythms of feces microbiota composition using 16S rRNA sequencing with the Illumina HiSeq PE250 high-throughput sequencing platform. Unqualified samples were eliminated for classification, resulting in the analysis of 86 feces samples (9 each from time points 1 (06:00), 6 (12:00), 8 (00:00), and 9 (06:00) and 10 each from the other time points). A total of 6,502,101 high-quality sequences were obtained after quality monitoring, with an average of 75,605 (range $46,014-87,152$ ) high-quality sequences per sample. The rarity curves of 86 samples (the minimum intercept at $97 \%$ sequence identity) were basically stable, indicating that the sampling depth was sufficient to describe the microbial community in the feces samples of laying hens.

When all other external conditions were the same, time of defecation could be among the most significant factors to explain the differences among individuals in microbiota community structure. The diversity of the feces microbiota community of laying hens was evaluated by the Observed ASVs and Chao1 and Shannon indexes (Figure 1). According to the results, community diversity (Observed ASVs and Chao1 and Shannon indexes) fluctuated significantly at different time points. The Observed ASVs and Chao1 index of the feces microbiota community of laying hens decreased gradually from 6:00 am on the first day to 12:00 noon on the second day but increased slowly from 12:00 midnight to 6:00 am on the third day (Figures 1A and 1B). Over time, the Shannon index also showed a trend of decreasing initially and then increasing slowly (Figure 1C). In addition, the alpha diversity of the feces microbiota at 6:00 am on the first day was significantly higher than that at other time points. To compare the overall differences in the feces microbiota composition of laying hens at 9 different time points, we conducted PCoA. The PCoA diagram shows that time points 1-4 are clustered together, time point 5 is separated, time points 6,7 and 8 are further separated and clustered together, and time point 9 is clustered together with time points 1-4 (Figure 1D). Although some samples overlapped among the nine groups, there were moderate differences among the nine different groups in the microbiota. The results showed that the composition of the microbiota oscillated periodically with time.

\section{Feces Microbiota Taxonomic Composition}

A GraPhIAn phylogenetic tree shows the relative abundance of taxonomic groups from the phylum to species level with the top 150 features (Figure 2A). There were six phylum-level taxonomic groups with high relative abundance, namely, Firmicutes, Proteobacteria, Bacteroidetes, Acidobacteria, Fusobacteria, and Actinobacteria, and their average relative abundances accounted for $57.88,14.11,11.26,6.23,3.62$, and $1.84 \%$ of the total sequences, respectively, which were regarded as the predominant bacterial phyla because their mean relative abundances accounted for greater than $1 \%$ of the total sequences. Notably, 
Firmicutes and Proteobacteria were the most abundant bacteria in the feces microbiota community of laying hens.

A total of 34 identified taxonomic groups were observed in the feces microbiota community at the family level, and 12 of the most predominant bacterial populations were present, including Turicibacteraceae, Streptococcaceae, Enterococcaceae, Lactobacillaceae, Clostridiaceae, Veillonellaceae,

Peptostreptococcaceae, Ruminococcaceae, Lachnospiraceae, Bacteroidaceae, Enterobacteriaceae, and Fusobacteriaceae, while their mean relative abundance exceeded $1 \%$ of the total sequences, and all these major bacterial families accounted for $43.76 \%$ of the total sequences in the feces microbiota of laying hens. The remaining bacterial families, including Erysipelotrichaceae, S24-7, Paraprevotellaceae, Prevotellaceae, Rikenellaceae, Porphyromonadaceae, Burkholderiaceae, Alcaligenaceae, Comamonadaceae, Hydrogenophilaceae, Pasteurellaceae, Moraxellaceae, Bradyrhizobiaceae, Caulobacteraceae, Helicobacteraceae, Syntrophobacteraceae, Koribacteraceae, Bifidobacteriaceae, Actinomycetaceae, Coriobacteriaceae, Nitrospiraceae and Methanobacteriaceae, were considered lowabundance bacterial families, while their sequences all accounted for $<1 \%$ of the total sequences, and they accounted for only $24.91 \%$ of the total sequences in the feces samples. Lactobacillaceae $(7.95 \%)$ belongs to the Firmicutes phylum, which was the most abundant classification group in the feces community of laying hens and was the most dominant family in the feces bacterial communities. The family-level taxonomic groups of the Firmicutes phylum were Turicibacteraceae, Streptococcaceae, Enterococcaceae, Lactobacillaceae, Clostridiaceae, Veillonellaceae, Peptostreptococcaceae, Ruminococcaceae, and Lachnospiraceae. Classified taxa in the Proteobacteria, Bacteroidetes, and Fusobacteria phyla at the family level were Bacteroidaceae, Enterobacteriaceae, and Fusobacteriaceae, respectively.

Further analysis of the data was performed to confirm whether the feces microbiota showed a circadian rhythm; therefore, box and line maps were made for eight bacterial phyla of the feces microbiota of laying hens at the phylum level, showing the relative abundances of the predominant phyla. The results showed that the relative abundance of the two most dominant phyla had an obvious circadian rhythm, which oscillated in antiphase. Highly robust circadian fluctuations were found, for instance, in Firmicutes and Proteobacteria, which had opposite fluctuations (Figure 2B and 2C). Between them, the relative abundance of Firmicutes reached its peak while that of Proteobacteria reached its trough at 06:00 the next morning. In addition, the change trend in the relative abundances of the other six predominant phyla did not fluctuate greatly (Additional file 1: Figure S1).

The 16S rRNA data of all feces samples were analyzed using the RF algorithm to determine the most important ASVs, and the top 30 important ASVs were Sutterella, Ruminococcus gnavus, Faecalibacterium, Lachnospiraceae, Ruminococcaceae, Enterococcus cecorum, Enterococcus, Lactobacillus reuteri, Lactobacillus, SMB53, Fusobacteriaceae, Clostridium colinum, Oscillospira, Bradyrhizobiaceae, JG37-AG-70, Burkholderia bryophila, S24-7, Porphyromonadaceae, Clostridium, Clostridiales, Megamonas, Salinispora tropica, Planococcaceae, Enterobacteriaceae, and ABS-6. These bacteria were classified into the phyla AD3, Bacteroidetes, Firmicutes, Fusobacteria, Nitrospirae, and Proteobacteria 
(Figure $3 A$ ). $A B S-6$ was the taxa related to $A D 3$ in the feces of laying hens with the lowest importance. Two ASVs, S24-7 and Porphyromonadaceae, were related to Bacteroidetes. Firmicutes was the most abundant, including Ruminococcus gnavus, Faecalibacterium, Lachnospiraceae, Ruminococcaceae, Enterococcus cecorum, Enterococcus, Lactobacillus reuteri, Lactobacillus, SMB53, Clostridium colinum, Oscillospira, Clostridium, Clostridiales, Megamonas, and Planococcaceae. Fusobacteria contains Fusobacteriaceae, and Nitrospirae contains JG37-AG-70. Five ASVs, including Sutterella, Bradyrhizobiaceae, Burkholderia bryophila, Salinispora tropica, and Enterobacteriaceae, were related to Proteobacteria, with the importance of Sutterella being the highest. This result was a rather remarkable outcome showing that eight ASVs, Ruminococcus gnavus, Faecalibacterium, Enterococcus cecorum, Ruminococcaceae, Lachnospiraceae, Clostridium, Clostridiales, and Megamonas, which exhibited a circadian rhythm, had displayed a steady rise initially and then a slight decrease in the relative abundance (Figure 3B-I). We found that the relative abundances of these bacteria, including Ruminococcus gnavus, Faecalibacterium, Lachnospiraceae, Clostridiales, and Megamonas, reached their peak at the same time point 4, while the peaks of other bacteria, including Enterococcus cecorum, Ruminococcaceae and Clostridium, were delayed. The remaining ASVs remained in a stable state (Additional file 1: Figure S2). Although the composition of the microbiota was quite different, the common core bacterial community in the feces may play an important role.

\section{Turnover of total, abundant, and rare of ASVs fractions}

We calculated $\beta N T I, R_{\text {Bray }}$ to determine the assembly processes driving circadian rhythm of microbiota community composition. Whether for intra-group samples or inter-group samples, the most $\beta N T I$ values between different samples were <-2 in the total ASVs, except ZT48 time point, suggesting the deterministric process; that is, homogeneous selection played a key role in shaping microbial composition in this study. Conversely, in the abundant and rare ASVs fractions, most $\beta N T I$ values were between -2 and 2, indicating that the stochastic process was important, except the ZT48 at rare ASVs was $>2$ (indicates variable selection). The $\mathrm{RC}_{\text {Bray }}$ values of the microbial communities in the abundant ASVs were found to be $>0.95$, refelcting the dispersal limitation (weak selection) dominantly determined the microbial community. However, RCBray values of the microbial communities in the rare ASVs were found to be $<0.95$, indicating the "non-dominant" fractions (Figure 4).

\section{Predicted Molecular Functions of Feces Microbiota}

We analyzed the composition of enzymes at level 3, and the top 30 important enzymes were selected, which were ranked according to the importance from highest to lowest in the feces samples (Figure 5A). These profiles revealed that all enzymes at level 3 were related to metabolism. In the KEGG database, hydroxydechloroatrazine ethylaminohydrolase was the most predominant enzyme related to xenobiotic biodegradation and metabolism at level 2. At level 2, carbohydrate metabolism was related to eight enzymes: methylaspartate ammonia-lyase, methylaspartate mutase, L-ribulose-5-phosphate 3-epimerase, D-glucosaminate-6-phosphate ammonia lyase, L-xylulokinase, homocitrate synthase, UDPgalactopyranose mutase, and methylmalonyl-CoA carboxytransferase. In addition, 
$\mathrm{N}$-carbamoylsarcosine amidase, homocitrate synthase and urocanate reductase were related to amino acid metabolism. The five enzymes methylaspartate mutase, urocanate reductase, caffeoyl-CoA 0-methyltransferase, D-glucosaminate-6-phosphate ammonia lyase, and methylaspartate ammonialyase gained rhythmicity upon metabolic activity of the feces microbiota (Figure 5B and 5C). In addition, there was no obvious circadian rhythmicity in the remaining enzymes (Additional file 1: Figure S3). In further analysis of the data, we selected the related microbiota according to their contribution to these 30 enzymes (Additional file 1: Figure S4). We found that Fusobacteriaceae, Lactobacillaceae and Enterobacteriaceae had a major contribution to these enzymes. In addition, Burkholderiaceae, Clostridiaceae, Bradyrhizobiaceae and Veillonellaceae were also involved in the contribution to these enzymes.

The differential abundance of the feces microbiota gave rise to different functions of the microbiota. To understand the development of the functions of the feces microbiota community over time, Matacyc pathway compositions of the feces microbiota community were predicted using PICRUSt based on 16S rRNA data (Figure 6A). Moreover, the predictable functions were sorted according to the importance from highest to lowest. The 30 most symbolic Matacyc pathways that had been annotated at level 3 were identified in the feces samples, including L-glutamate degradation VIII (to propanoate); creatinine degradation II; methylaspartate cycle; aerobactin biosynthesis; sulfoglycolysis; enterobacterial common antigen biosynthesis; L-glutamate degradation V (via hydroxyglutarate); phospholipases; superpathway of $L$-tryptophan biosynthesis; superpathway of $L$-arginine and $L$-ornithine degradation; superpathway of $\mathrm{L}$-arginine, putrescine, and 4-aminobutanoate degradation; adenosine nucleotides degradation IV; ppGpp biosynthesis; polyisoprenoid biosynthesis (Escherichiacoli); superpathway of sulfolactate degradation; $\operatorname{cob}(\mathrm{II})$ yrinate a,C-diamide biosynthesis I (early cobalt insertion); D-arabinose degradation III; superpathway of taurine degradation; superpathway of (Kdo)2-lipid A biosynthesis; reductive acetyl coenzyme A pathway; glutaryl-CoA degradation; L-lysine fermentation to acetate and butanoate; purine nucleobases degradation I (anaerobic); nylon-6 oligomer degradation; polymyxin resistance; superpathway of polyamine biosynthesis II; superpathway of hexuronide and hexuronate degradation; ethylmalonyl-CoA pathway; allantoin degradation IV (anaerobic); and superpathway of pyrimidine ribonucleotides de novo biosynthesis (Figure 6A). Interestingly, some of the Matacyc pathways fluctuated regularly over time. We found pathways involved in $\operatorname{cob}(\mathrm{II})$ yrinate a, c-diamide biosynthesis I (early cobalt insertion) to be among the microbiota functions oscillating (Figure 6B). Most remarkable and unexpected, however, were the functionalities that gained rhythmicity along with the microbiota, which included major pathways such as glutaryl-CoA degradation, L-glutamate degradation V (via hydroxyglutarate), L-lysine fermentation to acetate (Figure $6 \mathrm{C}$ ) and butanoate and L-glutamate degradation VIII (to propanoate) (Figure 6D), as exemplified by cob(II)yrinate a, c-diamide biosynthesis I (early cobalt insertion). In addition, there was no obvious circadian rhythmicity in the remaining pathways (Additional file 1: Figure S5). Taken together, these results suggested that there was an association between functionalities of the feces microbiota and passage of time.

\section{Co-occurrence Networks of Feces Bacteria}


To identify the potential interactions among the feces microbiota, cocorrelative network analysis was conducted for a feces bacterial community based on strong and significant correlations (Spearman's $r_{\mathrm{s}}<$ -0.5 or $r_{\mathrm{s}}>0.5, P<0.01$ ) (Figure 7). We performed a correlation analysis of the $30 \mathrm{ASV}$ s identified earlier and found that there were significant positive or negative correlations between them. The cocorrelative network of the feces microbiota consisted of 30 nodes (important bacteria). Five clusters (modules) were identified with high credibility in the bacterial cocorrelative network in feces. In this network, Sutterella had positive correlations with Faecalibacterium, Ruminococcus gnavus, Lachnospiraceae, Fusobacteriaceae, Oscillospira, SMB53, Clostridium, Clostridiales, Ruminococcaceae, Megamonas, Clostridium colinum, and JG37-AG-70. However, Sutterella had negative correlations with Enterobacteriaceae, Enterococcus, and Planococcaceae. In addition, Enterococcus had negative correlations with Faecalibacterium, Lachnospiraceae, Fusobacteriaceae, Ruminococcus gnavus, Oscillospira, Sutterella, Ruminococcaceae, Clostridiales, Clostridium colinum, and Megamonas but positive correlations with Enterobacteriaceae and Planococcaceae. The relative abundance of Lactobacillus showed positive correlations with Lactobacillus reuteri but negative correlations with JG37-AG-70 and ABS-6 (Figure 7).

\section{Discussion}

The present study was designed to demonstrate the diurnal oscillation of the feces microbiota. Using serial sampling, we described the phenomenon of circadian rhythmicity of the feces microbiota in laying hens. Alpha and beta diversity analyses indicated that temporal dynamic changes play an important role in the composition of the feces microbiota community. These results corroborate the findings of a large amount of previous work on circadian variations of the relative abundances in the feces microbiota in mouse stool samples [26-28, 39]. The diversity of the feces microbiota indicated that the microbiota community may overlap partially, but most were scattered, which is not entirely unexpected given the same age and diet of the subjects. As these differences are observed when the possible potential variable was photoperiod, rhythmic physiological process can directly affect feces microbiota composition. The secondary possibility is that the sampling time points were too dense to affect feeding behavior and stress.

We identified the two main phyla in the feces microbiota, Firmicutes and Proteobacteria, which was generally similar to previous results [40]. Although the relative abundance of the two kinds of bacteria had obvious circadian rhythms but fluctuated oppositely over $48 \mathrm{~h}$, the total ratio remained in a dynamic balance, which may indicate that there was a competitive relationship between the two kinds of bacteria. The same diurnal oscillation in the relative abundance of Firmicutes also appeared in our results [26, 28, 39]. However, the findings were contrary to previous studies on the feces microbiota of mouse stool samples, which suggested that no circadian rhythm was observed in the relative abundance of Proteobacteria [39], which might be related to the variety or diet of the experimental animals.

The 30 most important ASVs were selected using a RF model, and we found that temporal dynamic changes had a significant effect on the relative abundance of the important ASVs in the feces microbiota community, among which eight ASVs, Ruminococcus gnavus, Faecalibacterium, Ruminococcaceae, 
Enterococcus cecorum, Lachnospiraceae, Clostridium, Clostridiales, and Megamonas, gained cyclical fluctuations. Interestingly, one unexpected finding was the fact that all of these eight ASVs belonged to Firmicutes. Ruminococcus gnavus, gram-positive obligate anaerobes within the order Clostridiales [41], are involved in the decarboxylation of tryptophan to tryptamine [42], and there may be rhythmic fluctuations in this process. In addition, it was found that the relative abundance of Ruminococcus and Lactobacillus increased when the circadian rhythm of mice was disrupted [43]. Faecalibacterium, which belongs to the class Clostridia and the phylum Firmicutes and is a common gut microbe in chickens [44] and one of the main butyrate producers found in the gut [45], mainly ferments to produce short-chain fatty acids (SCFAs) [46] and has antiinflammatory properties to protect the intestine [47]. In addition, Faecalibacterium is involved in processes such as amino acid biosynthesis and starch and sugar degradation [48]. Studies have shown that increases in feces butyrate were found when the relative abundances of Lachnospiraceae, a family of digestive tract-associated bacteria [49], were increased [20]. In particular, the microbiota profile appears to favor butyrate production, a common indicator of gut health, potentially through increases in the members of Lachnospiraceae, Faecalibacterium and Ruminococcaceae $[20,45]$. Studies have found that high feather-pecking laying hens have a increased relative abundance of Clostridiales [14, 50], but the relative abundance of Lactobacillus was reduced compared to that in low feather-pecking laying hens. Previous studies have found that the circadian rhythm of the gut microbiota will be disordered and that the relative abundance of Clostridium will increase significantly in the small intestine during exposure to long-term darkness [51]. Megamonas, one of the major propionate producers, was antagonistic to Campylobacter persistence in the poultry gastrointestinal tract [52] and could encode enzymes involved in melibiose and alanine metabolism [53].

In our study, we found that the total ASVs were mainly driven by deterministric process, while stochastic process mainly dominated the abundant and rare ASVs. In addition, we also found that the rare ASVs were more ubiquitous than the abundant ASVs, which indicates that the abundant ASVs were driven by the time of feces excretion. This could be explained by the fact that rare ASVs occupied a favorable position, competitively utilized a broader array of resources than the abundant ASVs, and effectively adapted to the environment [38]. Another possible explanation for this was that the $\beta N T I$ and $\mathrm{RC}_{\mathrm{Bray}}$ values, which reflected that compared with the rare ASV fraction that was "non-dominant" fractions, the abundant ASV fraction was driven by a weaker selection. This may be due to the competition capacity and growth rate of the abundant ASVs were lower and therefore limited in the environmental breadths [54].

If poultry are exposed to light for more than $20 \mathrm{~h}$ and free to eat during the period, the activity of the gut microbiota and intestinal function are abnormal, while kept in the dark for at least six h every day, the circadian rhythm of poultry will be improved, especially in early life [32]. This finding shows that the fluctuation of the circadian rhythm can be controlled by factors such as food intake and light. A longer dark time can enhance the acquisition and proliferation of beneficial microbiota constituents, which ultimately improves immunity, reduces mortality, and potentially reduces the need for antibiotic treatment in poultry farming [32]. To maintain the activity and good production performance of laying hens, the 
illumination time is usually more than $16 \mathrm{~h}$ per day, and sufficient feed is always supplied. However, longterm free eating and limited activity space can easily lead to metabolic disorder of laying hens, which can lead to fatty liver and abdominal fat deposition, eventually reducing production performance and egg quality and increasing mortality. A previous study reported that disrupting the rhythm of the mouse diet can change the composition of the gut microbiota and significantly increase the main probiotics, such as Lactobacillus and Bifidobacterium, but reduce Helicobacter [55], while causing obesity and metabolic syndrome [56]. We speculate that feeding food in batches and shortening the illumination time to prolong the dark time and adopting intermittent illumination may be more conducive to a better circadian rhythm of the gut microbiota.

The circadian characteristics of the microbiota extend beyond its taxonomic composition and include its potential metabolic function, as well as the enzymes involved in physiological metabolism. The potential functions of the feces microbiota were determined using PICRUSt based on 16S rRNA data. The results showed that the most abundant functions, including xenobiotic biodegradation and metabolism, carbohydrate metabolism, and amino acid metabolism, were consistent with the metabolic functions of amino acids and carbohydrates, which are necessary for microbiota survival. Although the enzymes involved in the reaction had a significant effect on the most important microbiota functions at level 3 , some of them gained a circadian rhythm, including methylaspartate mutase, urocanate reductase, caffeoyl-CoA 0-methyltransferase, D-glucosaminate-6-phosphate ammonia-lyase, and methylaspartate ammonia-lyase. Further analysis indicated the related bacteria that contributed the most to these enzymes. Fusobacteriaceae, Lactobacillaceae and Enterobacteriaceae had a major contribution and were the most predominant bacteria at the family level in the feces microbiota. Prediction of the potential function of the feces microbiota showed that cob(II)yrinate a, c-diamide biosynthesis I (early cobalt insertion), glutaryl-CoA degradation, L-glutamate degradation $\mathrm{V}$ (via hydroxyglutarate), L-lysine fermentation to acetate, and butanoate and L-glutamate degradation VIII (to propanoate) gained temporal changes in bacterial functions, which gained rhythmicity upon physiological metabolism and supported a functional link between the circadian rhythm in the feces microbiota and metabolic homeostasis. The only reported glutaric acid catabolism was the dehydrogenation pathway of glutarylCoA [57]. Glutamate is a neurotransmitter that may be released from neurons or the gut microbiota [58], and its abundant presence is consistent with gut physiology and inflammation [59]. Its degradation may slow the inflammatory response. Most of these rhythmic pathways involve the degradation of amino acids and the production of SCFAs, such as acetic acid and propionic acid. It was found that the daily change in feces SCFA concentration was consistent with the rhythm of FFAR2/3 expression in the colonic muscularis [60]. SCFAs produced by microbiota fermentation may play an important role in maintaining circadian rhythm.

In our network analysis, Sutterella, Faecalibacterium, Ruminococcus gnavus, Fusobacteriaceae, Oscillospira, SMB53, Clostridium, Clostridiales, Ruminococcaceae, Lachnospiraceae, Megamonas, Clostridium colinum, and JG37-AG-70 demonstrated a positive correlation, potentially because all of them are SCFA-producing strains. Sutterella was the most important ASV in the feces microbiota in our results; however, previous studies have concluded that Sutterella species are possible proinflammatory agents 
and could be associated with the adhesion to intestinal epithelial cells and increased mucosal prevalence [61]. Lactobacillus spp. have various known benefits to metabolism and intestinal health, from antimicrobial activity to their probiotic activity $[62,63]$. The relative abundance of JG37-AG-70 was negatively correlated with Lactobacillus abundance but positively correlated with Clostridium abundance, which also verified the previous report that Lactobacillus was depleted when pathogens such as Clostridium were enriched [64]. Multiple studies have proven that intestinal inflammation is frequently accompanied by an imbalanced microbiota community, which is often characterized by a relative increase in facultative anaerobic Enterobacteriaceae [65]. The abundance of Enterobacteriaceae correlated with the magnitude of colitis [66]. The ubiquitous gram-positive bacterium Enterococcus is a symbiotic bacterium of the intestinal tract but can cause serious opportunistic infections [67-69]. The Planococcaceae family from the Firmicutes phylum accounts for $18 \%$ of the feces microbiota of cattle; therefore, it is considered a normal resident of the bovine gut and may be collected from viscera and carcasses [70,71]. Salinispora tropica, Burkholderia bryophila, and Enterococcus cecorum were the important bacteria in the feces microbiota; however, they did not play an important role in the network and were completely independent of the outside two clusters. These important bacteria may not be functional in the network because of the high diversity of the feces microbiota.

Generally, the results showed that the relative abundance of a small fraction of the total feces microbiota oscillated with a rhythm and that fewer still oscillated with a 24-h rhythm. Ten replicates per time point were used in our study, which is the same as mouse study results that have previously been reported [24, 26]. However, one of the main differences between mice and chickens is the applicability of feces samples to the study of the gut microbiota. Mouse feces samples are applicable to human health [72] and are an accepted and reliable source of information about the gut microbiota. However, chicken feces samples are not reliable indicators of the gut microbiota, as reported previously [73]. Together with the applicability of feces samples and the smaller space requirements, it is less challenging to conduct more reproducible longitudinal and temporal studies in mouse models than in chicken models. While our research reveals that the composition of only a part of the feces microbiota in laying hens that exhibits daily fluctuations, further confirmation of the circadian rhythm of the gut microbiota of laying hens and its functional mechanism will provide additional data.

\section{Conclusions}

This study set out to explore the circadian rhythm of the feces microbiota in laying hens, and we systematically characterized the feces microbiota in 86 samples obtained within $48 \mathrm{~h}$. The time of defecation could be among the important factors influencing the composition and diversity of the feces microbiota community. We also firstly systematically proposed the assembly pattern of the microbial community in the circadian thythm of the feces microbiota. Abundant ASVs were more shaped based on the disperal limitation (weak selection), whereas rare ASVs were the "nod-dominant" fraction. These results lay a foundation for further exploring the circadian rhythm of the gut microbiota in laying hens. 


\section{Abbreviations}

RF: random forest; ASVs: amplicon sequence variants; SCFAs: short-chain fatty acids

\section{Declarations}

\section{Ethics approval and consent to participate}

The experimental design and procedures were conducted according to the institutional guidelines for the care and use of experimental procedures involving animals were approved by the Animal Experimental Committee of South China Agricultural University (SYXK2014-0136). In this study, only fresh feces samples collected from anal swabs were analyzed, and no animals died or were injured.

\section{Consent for publication}

No applicable.

\section{Availability of data and materials}

The sequencing data in this study were deposited in the European Nucleotide Archive (ENA) under accession number PRJEB41206.

\section{Competing interests}

The authors declare that they have no competing interests.

\section{Funding}

This research was supported by the Basic Research Program Basic and Applied Basic Research Project of Guangzhou and the earmarked fund from Modern Agroindustry Technology Research System (CARS-40).

\section{Authors' contributions}

$Y Z, X D L$ and JDM conceived and designed the experiments. YZ, LS, SYZ, RZ, YW and YBW contributed to sampling. YZ LS and JDM performed the experiments and analyzed the data. YZ and JDM wrote the paper. All authors revised the paper thoroughly.

\section{Acknowledgments}

No applicable.

\section{Authors' information}

${ }^{1}$ College of Animal Science, National Engineering Research Center for Breeding swine Industry, South China Agricultural University, Guangzhou 510642, China; 
${ }^{2}$ Ministry of Agriculture Key Laboratory of Tropical Agricultural Environment, South China Agricultural University, Guangzhou 510642, China;

${ }^{3}$ Guangdong Provincial Key Lab of Agro-Animal Genomics and Molecular Breeding and Key Lab of Chicken Genetics, Breeding and Reproduction, Ministry of Agriculture, Guangzhou 510642, China.

\section{References}

1. Choi K. Y., Lee T. K., Sul W. J.: Metagenomic Analysis of Chicken Gut Microbiota for Improving Metabolism and Health of Chickens - A Review. Asian-Australas J Anim Sci. 28(9):1217-1225 (2015)

2. Gao P., Ma C., Sun Z. et al.: Feed-additive probiotics accelerate yet antibiotics delay intestinal microbiota maturation in broiler chicken. Microbiome. 5(1):91 (2017)

3. Li X., Cao Z. H., Yang Y. T. et al.: Correlation between Jejunal Microbial Diversity and Muscle Fatty Acids Deposition in Broilers Reared at Different Ambient Temperatures. Sci Rep-Uk. 9 (2019)

4. Henchion M., McCarthy M., Resconi V. C. et al.: Meat consumption: trends and quality matters. Meat Sci. 98(3):561-568 (2014)

5. Huang P., Zhang Y., Xiao K. et al.: The chicken gut metagenome and the modulatory effects of plantderived benzylisoquinoline alkaloids. Microbiome. 6(1):211 (2018)

6. Qiao H., Song Y., Shi H. et al.: Fermented Astragalus in diet altered the composition of fecal microbiota in broiler chickens. Amb Express. 8(1):151 (2018)

7. Wang Q. Q., Yates S. R.: Laboratory study of oxytetracycline degradation kinetics in animal manure and soil. J Agr Food Chem. 56(5):1683-1688 (2008)

8. Hu X. G., Zhou Q. X., Luo Y.: Occurrence and source analysis of typical veterinary antibiotics in manure, soil, vegetables and groundwater from organic vegetable bases, northern China. Environmental Pollution. 158(9):2992-2998 (2010)

9. Sommer M. O. A., Dantas G., Church G. M.: Functional characterization of the antibiotic resistance reservoir in the human microflora. Science. 325(5944):1128-1131 (2009)

10. Zhang Y., Liu Z., Liu R. et al.: Alteration of Hepatic Gene Expression along with the Inherited Phenotype of Acquired Fatty Liver in Chicken. Genes (Basel). 9(4) (2018)

11. Chen L., Zhang T., Zhang S. S. et al.: Identification of Long Non-Coding RNA-Associated Competing Endogenous RNA Network in the Differentiation of Chicken Preadipocytes. Genes-Basel. 10(10) (2019)

12. Geng Y., Ma Q., Wang Z. et al.: Dietary vitamin D3 supplementation protects laying hens against lipopolysaccharide-induced immunological stress. Nutr Metab (Lond). 15:58 (2018)

13. Bestman M. W. P., Wagenaar J. P.: Farm level factors associated with feather pecking in organic laying hens. Livest Prod Sci. 80(1-2):133-140 (2003)

14. Birkl P., Bharwani A., Kjaer J. B. et al.: Differences in cecal microbiome of selected high and low feather-pecking laying hens. Poultry Sci. 97(9):3009-3014 (2018) 
15. Ridaura V. K., Faith J. J., Rey F. E. et al.: Gut Microbiota from Twins Discordant for Obesity Modulate Metabolism in Mice. Science. 341(6150):1079-U1049 (2013)

16. Turnbaugh P. J., Ley R. E., Mahowald M. A. et al.: An obesity-associated gut microbiome with increased capacity for energy harvest. Nature. 444(7122):1027-1031 (2006)

17. Valdes A. M., Walter L., Segal E. et al.: Role of the gut microbiota in nutrition and health. Bmj-Brit Med J. 361 (2018)

18. Cao Z. P., Wang X. Y., Pang Y. et al.: Biointerfacial self-assembly generates lipid membrane coated bacteria for enhanced oral delivery and treatment. Nat Commun. 10 (2019)

19. Huttenhower C., Gevers D., Knight R. et al.: Structure, function and diversity of the healthy human microbiome. Nature. 486(7402):207-214 (2012)

20. Estaki M., Pither J., Baumeister P. et al.: Cardiorespiratory fitness as a predictor of intestinal microbial diversity and distinct metagenomic functions. Microbiome. 4 (2016)

21. Parkar S. G., Kalsbeek A., Cheeseman J. F.: Potential Role for the Gut Microbiota in Modulating Host Circadian Rhythms and Metabolic Health. Microorganisms. 7(2) (2019)

22. Chan M. C., Spieth P. M., Quinn K. et al.: Circadian rhythms: From basic mechanisms to the intensive care unit. Crit Care Med. 40(1):246-253 (2012)

23. Thaiss C. A., Zeevi D., Levy M. et al.: A day in the life of the meta-organism: diurnal rhythms of the intestinal microbiome and its host. Gut Microbes. 6(2):137-142 (2015)

24. Thaiss C. A., Levy M., Korem T. et al.: Microbiota Diumal Rhythmicity Programs Host Transcriptome Oscillations. Cell. 167(6):1495-+ (2016)

25. Hut R. A., Beersma D. G.: Evolution of time-keeping mechanisms: early emergence and adaptation to photoperiod. Philos Trans R Soc Lond B Biol Sci. 366(1574):2141-2154 (2011)

26. Thaiss C. A., Zeevi D., Levy M. et al.: Transkingdom Control of Microbiota Diurnal Oscillations Promotes Metabolic Homeostasis. Cell. 159(3):514-529 (2014)

27. Leone V., Gibbons S. M., Martinez K. et al.: Effects of Diurnal Variation of Gut Microbes and High-Fat Feeding on Host Circadian Clock Function and Metabolism. Cell Host Microbe. 17(5):681-689 (2015)

28. Zarrinpar A., Chaix A., Yooseph S. et al.: Diet and Feeding Pattern Affect the Diurnal Dynamics of the Gut Microbiome. Cell Metab. 20(6):1006-1017 (2014)

29. Cribbet M. R., Logan R. W., Edwards M. D. et al.: Circadian rhythms and metabolism: from the brain to the gut and back again. Annals Reports, Vol 1385. 1385:21-40 (2016)

30. Wang J., Mauvoisin D., Martin E. et al.: Nuclear Proteomics Uncovers Diurnal Regulatory Landscapes in Mouse Liver. Cell Metab. 25(1):102-117 (2017)

31. Farghly M. F. A., Mahrose K. M., Rehman Z. U. et al.: Intermittent lighting regime as a tool to enhance egg production and eggshell thickness in Rhode Island Red laying hens. Poultry Sci. 98(6):2459-2465 (2019)

32. Hieke A. S. C., Hubert S. M., Athrey G.: Circadian disruption and divergent microbiota acquisition under extended photoperiod regimens in chicken. Peerj. 7 (2019) 
33. Arumugam M., Raes J., Pelletier E. et al.: Enterotypes of the human gut microbiome. Nature. 473(7346):174-180 (2011)

34. Hadizadeh F., Walter S., Belheouane M. et al.: Stool frequency is associated with gut microbiota composition. Gut. 66(3):559-560 (2017)

35. Bokulich N. A., Joseph C. M. L., Allen G. et al.: Next-Generation Sequencing Reveals Significant Bacterial Diversity of Botrytized Wine. Plos One. 7(5) (2012)

36. Stegen J. C., Lin X., Konopka A. E. et al.: Stochastic and deterministic assembly processes in subsurface microbial communities. ISME J. 6(9):1653-1664 (2012)

37. Jiang Y., Huang H., Tian Y. et al.: Stochasticity versus determinism: Microbial community assembly patterns under specific conditions in petrochemical activated sludge. Journal of Hazardous Materials. 407 (2021)

38. Jiao S., Lu Y.: Soil pH and temperature regulate assembly processes of abundant and rare bacterial communities in agricultural ecosystems. Environ Microbiol. 22(3):1052-1065 (2020)

39. Liang X., Bushman F. D., FitzGerald G. A.: Rhythmicity of the intestinal microbiota is regulated by gender and the host circadian clock. P Natl Acad Sci USA. 112(33):10479-10484 (2015)

40. Zhu L. H., Liao R. R., Wu N. et al.: Heat stress mediates changes in fecal microbiome and functional pathways of laying hens. Appl Microbiol Biot. 103(1):461-472 (2019)

41. Chua H. H., Chou H. C., Tung Y. L. et al.: Intestinal Dysbiosis Featuring Abundance of Ruminococcus gnavus Associates With Allergic Diseases in Infants. Gastroenterology. 154(1):154-167 (2018)

42. Gao J., Xu K., Liu H. N. et al.: Impact of the Gut Microbiota on Intestinal Immunity Mediated by Tryptophan Metabolism. Front Cell Infect Mi. 8 (2018)

43. Deaver J. A., Eum S. Y., Toborek M.: Circadian Disruption Changes Gut Microbiome Taxa and Functional Gene Composition. Front Microbiol. 9 (2018)

44. Oakley B. B., Lillehoj H. S., Kogut M. H. et al.: The chicken gastrointestinal microbiome. Fems Microbiol Lett. 360(2):100-112 (2014)

45. Duncan S. H., Hold G. L., Harmsen H. J. M. et al.: Growth requirements and fermentation products of Fusobacterium prausnitzii, and a proposal to reclassify it as Faecalibacterium prausnitzii gen. nov., comb. nov. Int J Syst Evol Micr. 52:2141-2146 (2002)

46. Riviere A., Selak M., Lantin D. et al.: Bifidobacteria and Butyrate-Producing Colon Bacteria: Importance and Strategies for Their Stimulation in the Human Gut. Front Microbiol. 7 (2016)

47. Sartor R. B.: Genetics and Environmental Interactions Shape the Intestinal Microbiome to Promote Inflammatory Bowel Disease Versus Mucosal Homeostasis. Gastroenterology. 139(6):1816-1819 (2010)

48. Amato K. R., Mallott E. K., McDonald D. et al.: Convergence of human and Old World monkey gut microbiomes demonstrates the importance of human ecology over phylogeny. Genome Biol. 20(1) (2019) 
49. Arvonen M., Vanni P., Sarangi A. N. et al.: Microbial orchestra in juvenile idiopathic arthritis: Sounds of disarray? Immunol Rev. 294(1):9-26 (2020)

50. van der Eijk J. A. J., de Vries H., Kjaer J. B. et al.: Differences in gut microbiota composition of laying hen lines divergently selected on feather pecking. Poultry Sci. 98(12):7009-7021 (2019)

51. Wu G. Y., Tang W. L., He Y. et al.: Light exposure influences the diurnal oscillation of gut microbiota in mice. Biochemical and Biophysical Research Communications. 501(1):16-23 (2018)

52. Scupham A. J., Jones J. A., Rettedal E. A. et al.: Antibiotic Manipulation of Intestinal Microbiota To Identify Microbes Associated with Campylobacter jejuni Exclusion in Poultry. Appl Environ Microb. 76(24):8026-8032 (2010)

53. Polansky O., Sekelova Z., Faldynova M. et al.: Important Metabolic Pathways and Biological Processes Expressed by Chicken Cecal Microbiota. Appl Environ Microb. 82(5):1569-1576 (2016)

54. Jousset A., Bienhold C., Chatzinotas A. et al.: Where less may be more: how the rare biosphere pulls ecosystems strings. ISME J. 11(4):853-862 (2017)

55. Wang S., Huang M. Q., You X. et al.: Gut microbiota mediates the anti-obesity effect of calorie restriction in mice. Sci Rep-Uk. 8 (2018)

56. Chaix A., Lin T., Le H. D. et al.: Time-Restricted Feeding Prevents Obesity and Metabolic Syndrome in Mice Lacking a Circadian Clock. Cell Metab. 29(2):303-+ (2019)

57. Zhang M. M., Gao C., Guo X. T. et al.: Increased glutarate production by blocking the glutaryl-CoA dehydrogenation pathway and a catabolic pathway involving L-2-hydroxyglutarate. Nat Commun. 9 (2018)

58. Mazzoli R., Pessione E.: The Neuro-endocrinological Role of Microbial Glutamate and GABA Signaling. Front Microbiol. 7 (2016)

59. Davies L. C., Rice C. M., Palmieri E. M. et al.: Peritoneal tissue-resident macrophages are metabolically poised to engage microbes using tissue-niche fuels. Nat Commun. 8 (2017)

60. Segers A., Desmet L., Thijs T. et al.: The circadian clock regulates the diurnal levels of microbial shortchain fatty acids and their rhythmic effects on colon contractility in mice. Acta Physiol. 225(3) (2019)

61. Hiippala K., Kainulainen V., Kalliomaki M. et al.: Mucosal Prevalence and Interactions with the Epithelium Indicate Commensalism of Sutterella spp. Front Microbiol. 7 (2016)

62. Marco M. L., Heeney D., Binda S. et al.: Health benefits of fermented foods: microbiota and beyond. Curr Opin Biotech. 44:94-102 (2017)

63. Patten D. A., Laws A. P.: Lactobacillus-produced exopolysaccharides and their potential health benefits: a review. Benef Microbes. 6(4):457-471 (2015)

64. Shaufi M. A. M., Sieo C. C., Chong C. W. et al.: Deciphering chicken gut microbial dynamics based on high-throughput 16S rRNA metagenomics analyses. Gut Pathog. 7 (2015)

65. Nagalingam N. A., Lynch S. V.: Role of the microbiota in inflammatory bowel diseases. Inflamm Bowel Dis. 18(5):968-980 (2012) 
66. Huang Y. L., Chassard C., Hausmann M. et al.: Sialic acid catabolism drives intestinal inflammation and microbial dysbiosis in mice. Nat Commun. 6 (2015)

67. d'Herouel A. F., Wessner F., Halpern D. et al.: A simple and efficient method to search for selected primary transcripts: non-coding and antisense RNAs in the human pathogen Enterococcus faecalis. Nucleic Acids Res. 39(7):E46-U88 (2011)

68. Gilmore M. S., Ferretti J. J.: Microbiology: The thin line between gut commensal and pathogen. Science. 299(5615):1999-+ (2003)

69. Daw K., Baghdayan A. S., Awasthi S. et al.: Biofilm and planktonic Enterococcus faecalis elicit different responses from host phagocytes in vitro. Fems Immunol Med Mic. 65(2):270-282 (2012)

70. Mao S. Y., Zhang R. Y., Wang D. S. et al.: The diversity of the fecal bacterial community and its relationship with the concentration of volatile fatty acids in the feces during subacute rumen acidosis in dairy cows. Bmc Vet Res. 8 (2012)

71. Korsak N., Taminiau B., Hupperts C. et al.: Assessment of bacterial superficial contamination in classical or ritually slaughtered cattle using metagenetics and microbiological analysis. Int J Food Microbiol. 247:79-86 (2017)

72. Nguyen T. L. A., Vieira-Silva S., Liston A. et al.: How informative is the mouse for human gut microbiota research? Dis Model Mech. 8(1):1-16 (2015)

73. Stanley D., Geier M. S., Chen H. et al.: Comparison of fecal and cecal microbiotas reveals qualitative similarities but quantitative differences. Bmc Microbiol. 15 (2015)

\section{Figures}


A

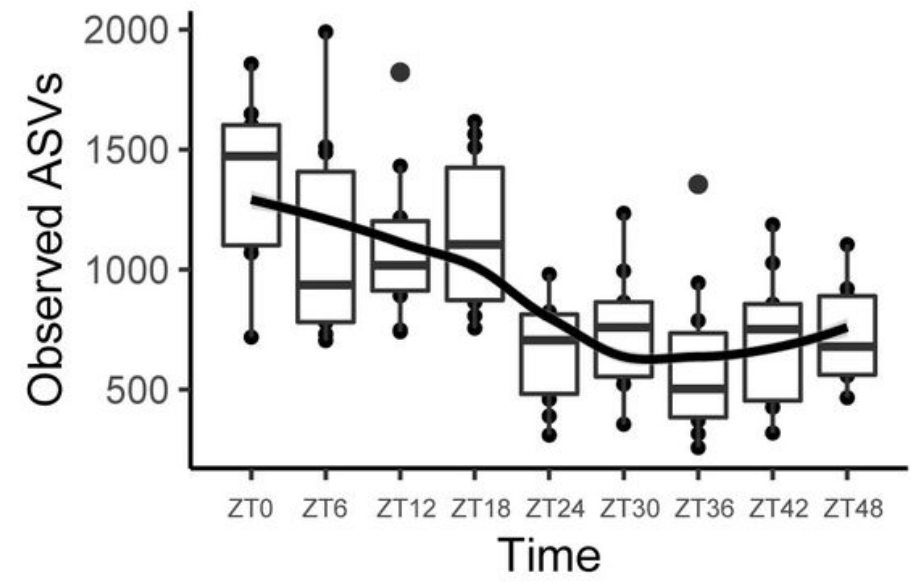

C

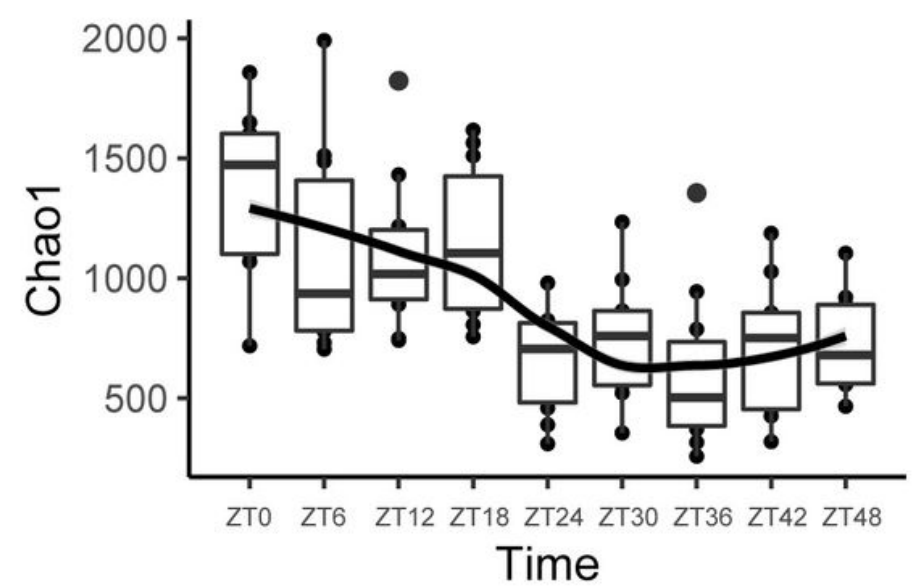

B

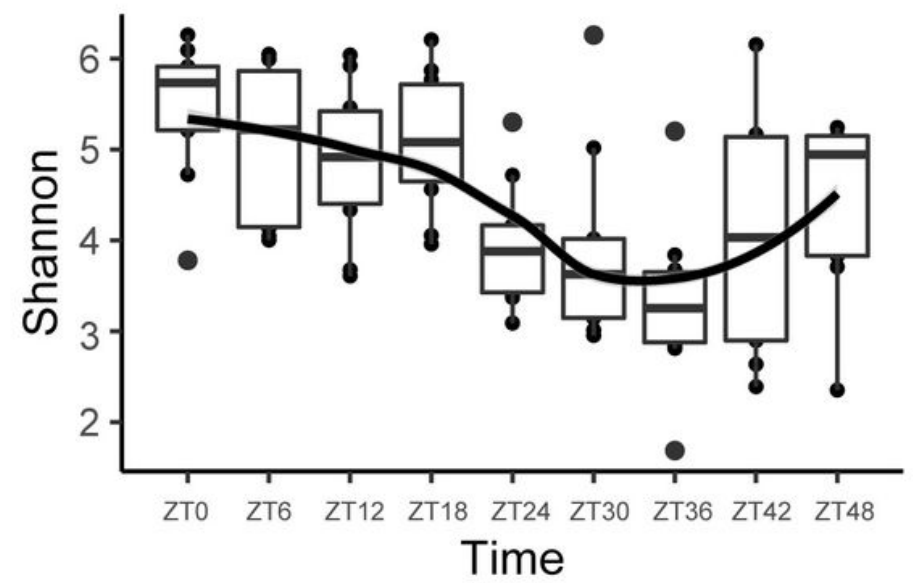

$\mathrm{D}$

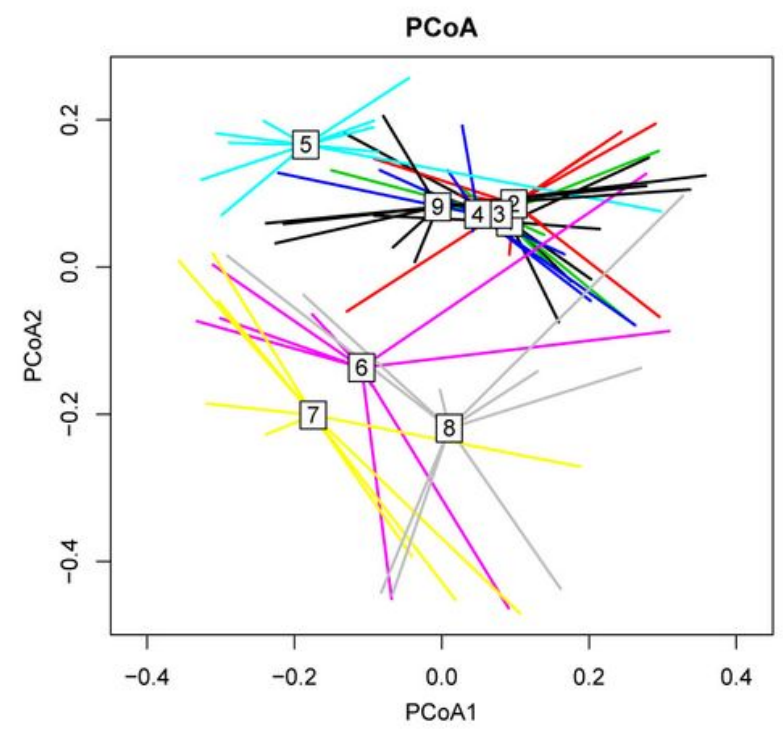

Figure 1

Changes in feces microbiota diversity. (A) Bacterial alpha diversity determined by the Observed ASVs; (B) Bacterial alpha diversity determined by the Chao1 index; (C) Bacterial alpha diversity determined by the Shannon index; (D) PCoA of the feces microbiota at each time point. 

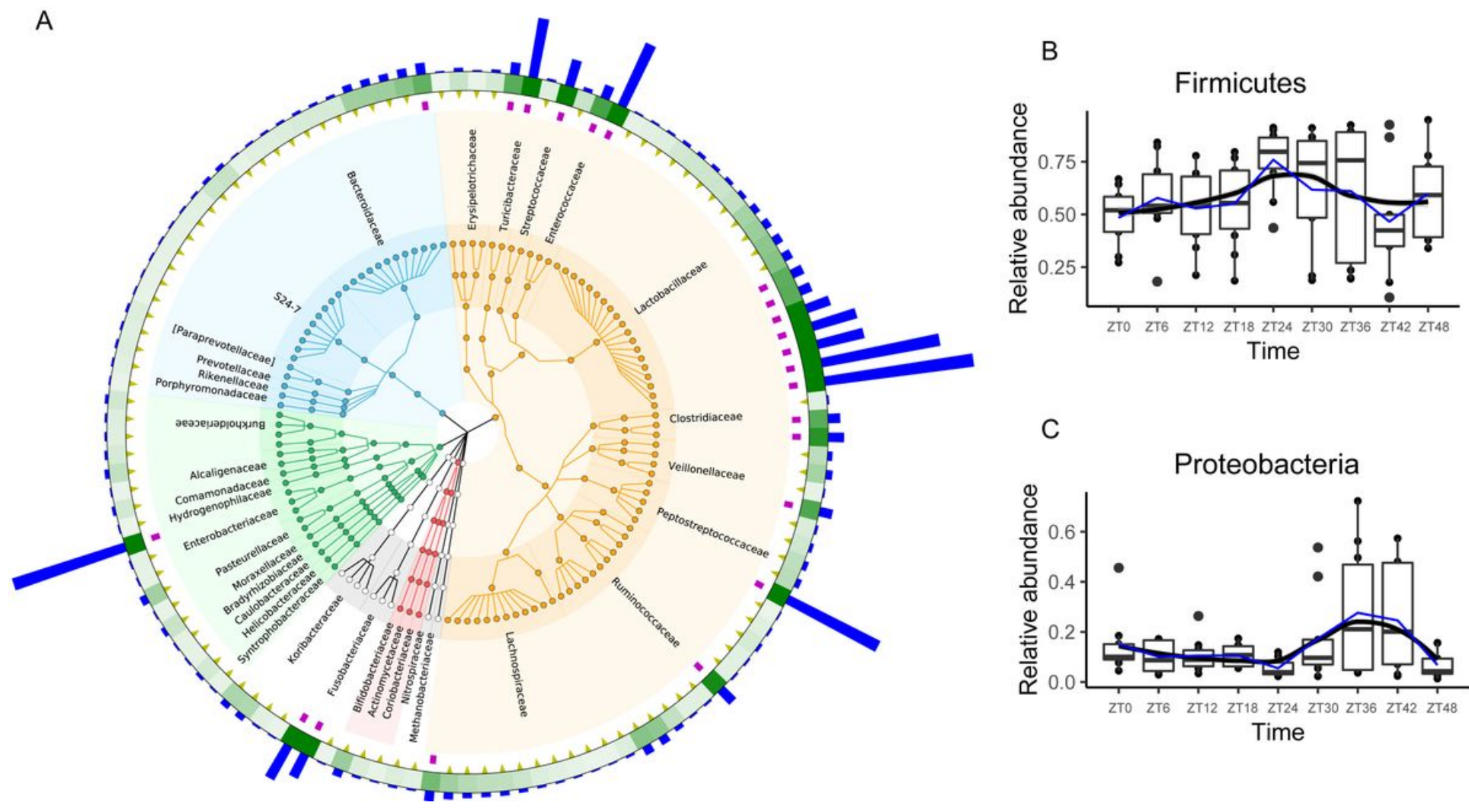

\section{Figure 2}

Different bacterial compositions based on the 16S rRNA data analysis. (A) GraPhlAn phylogenetic tree analysis of the feces microbiota from the phylum to species level; (B-C) The temporal changes in the relative abundances of predominant bacteria (Firmicutes and Proteobacteria) at the phylum level. 

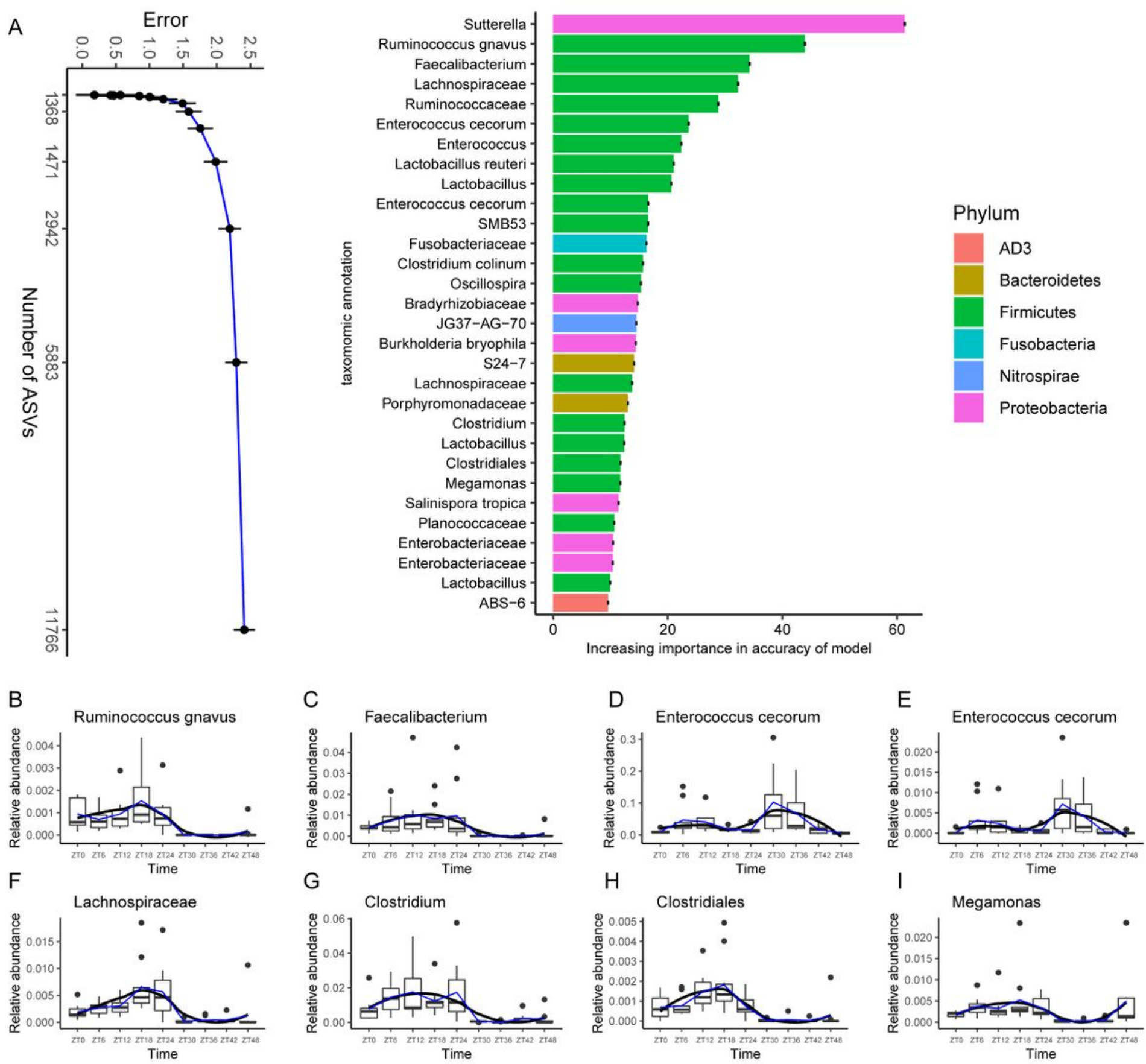

Figure 3

The most abundant bacteria identified by random forest analysis. (A) Tracing the source of the top 30 most abundant bacteria in the feces microbiota; (B-I) The temporal changes in the relative abundance of the eight most important bacteria. 

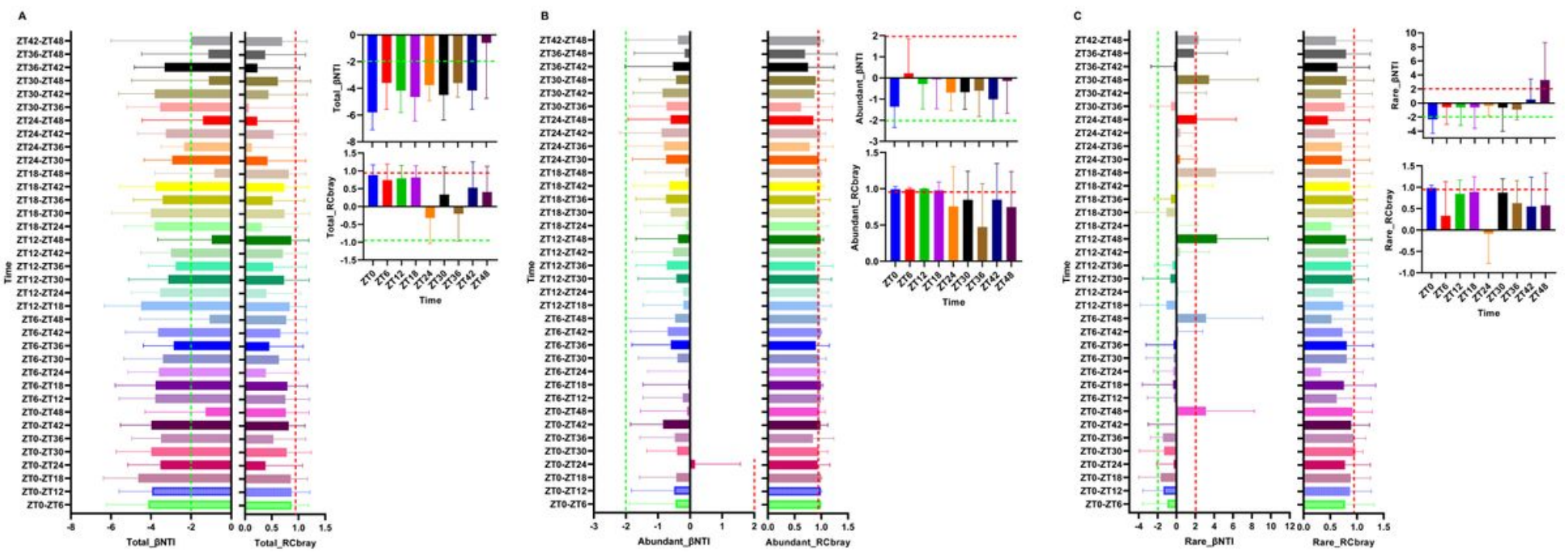

Figure 4

The Beta Nearest Taxon Index ( $\beta N T I)$ of the total $(A)$, abundant $(B)$, and rare $(C)$ bacterial communities and the Raup-Crick metric based on relative abundances of the ASVs (RCBray). The relative abundance of ASVs $\geq 1 \%$ and $<0.1 \%$ were assigned as abundant ASVs and rare ASVs, respectively.
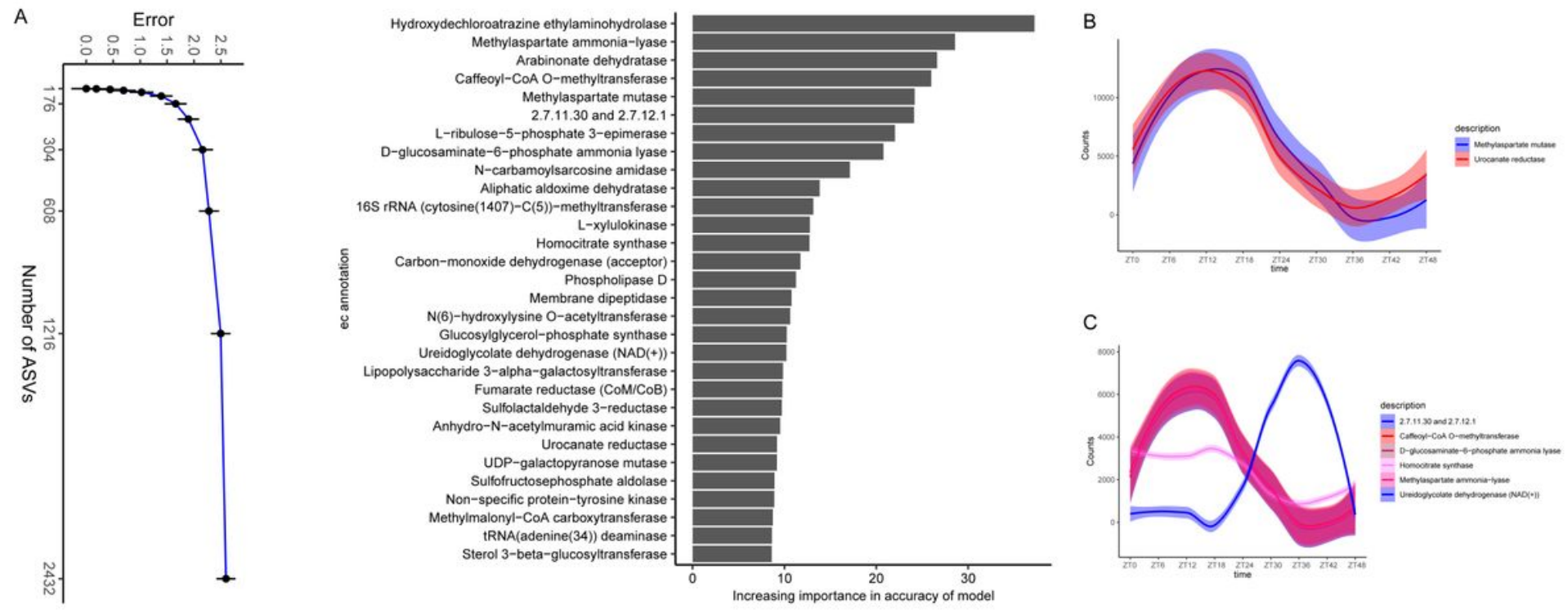

\section{Figure 5}

The most abundant enzymes identified by random forest analysis. (A) Tracing the source of the top 30 most abundant enzymes in the feces microbiota; (B-C) The temporal changes in the contributing enzymes. 

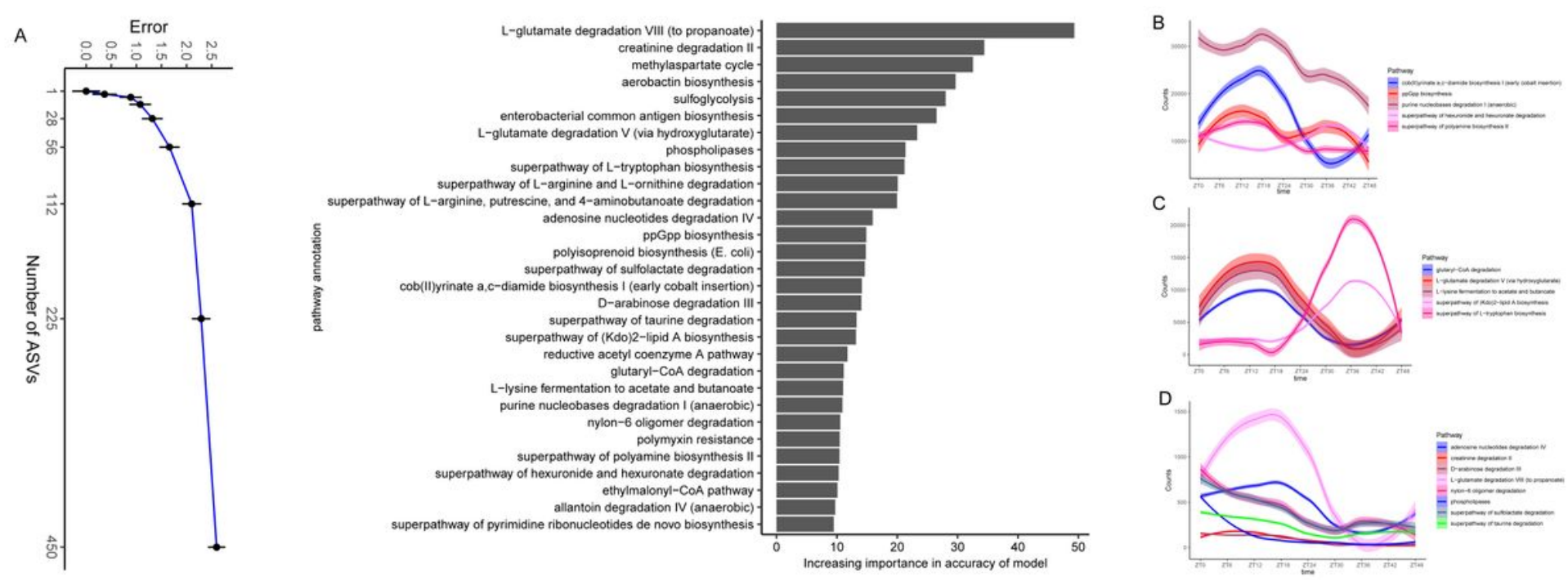

Figure 6

The important bacterial functions identified by random forest analysis. (A) Tracing the source of the top 30 most important bacterial functions in the feces microbiota; (B-D) The temporal changes in the contributing bacterial functions.

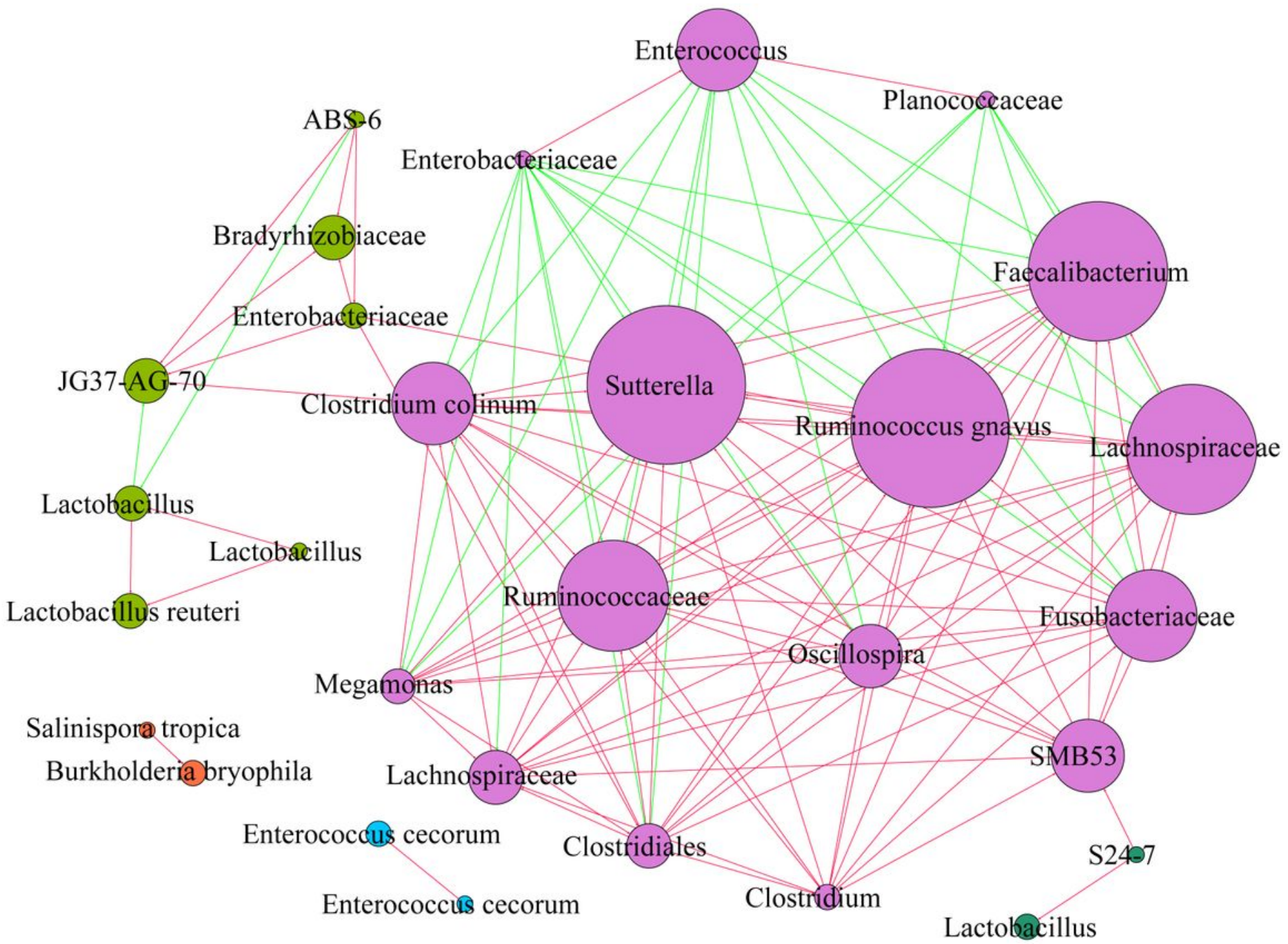




\section{Figure 7}

Interaction network of cooccurring important genera within feces bacteria in laying hens $(n=86)$. The node represents the predominant genera in laying he ns feces, the size of each node represents the relative abundance, and the color of the node represents the module. The edges represent negative (red) or positive (green) correlations of two connected nodes.

\section{Supplementary Files}

This is a list of supplementary files associated with this preprint. Click to download.

- FigureS1.pdf

- Figures2.pdf

- Figures3.pdf

- Figures4.pdf

- Figures5.pdf

- TableS1.docx 